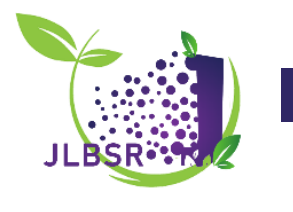

\title{
Biofilm Forming Potential of Escherichia coli from Various Sources
}

\author{
Kome Otokunefor ${ }^{1, *}$, Deborah E. Melex ${ }^{1}$, Gideon O. Abu ${ }^{2}$ \\ ${ }^{1}$ Department of Microbiology, Faculty of Science, University of Port Harcourt. PMB 5323 Port Harcourt, Nigeria, \\ (kome.otokunefor@uniport.edu.ng) \\ ${ }^{2}$ Department of Microbiology, Faculty of Science, University of Port Harcourt. PMB 5323 Port Harcourt, Nigeria, \\ (gideon.abu@uniport.edu.ng) \\ *Correspondence: kome.otokunefor@uniport.edu.ng
}

\begin{abstract}
Majority of bacterial communities exist as biofilms and these contribute to the survival of the bacteria. Biofilm development has been associated with protection from adverse environmental conditions and resistance to harmful agents. Generally, however data on biofilm-forming potential of bacteria in Nigeria is sparse. This study was therefore aimed at analyzing variations in biofilm-forming potential of Escherichia coli from various sources in Port Harcourt, Nigeria. Previously characterized clinical (30) and non-clinical (30) $E$. coli isolates were assessed for their biofilm-forming potential using the Congo Red agar method and variations in this potential determined as weak, moderate or strong. Majority of isolates $(67 \%)$ had the potential to form biofilms but only $40 \%$ of isolates exhibiting biofilm-forming potential were from clinical sources. Isolates exhibited variable degrees of biofilm-forming potential, with only non-clinical isolates exhibiting strong potential. Majority of both clinical and non-clinical isolates $(68.7 \%$ and $88 \%$ respectively) exhibited moderate biofilm-forming potential. The higher occurrence of $E$. coli exhibiting biofilm-forming potential among non-clinical isolates possibly reflects the essential role biofilms play in the survival of bacteria in nature, but not in infection cases. This study reports on a high level association between the isolates and biofilm production and highlights differences in the abilities of biofilm production between clinical and non-clinical isolates.
\end{abstract}

Keywords: Biofilm Potential, Escherichia coli, Congo Red Agar Method, Clinical vs Non-clinical

Received: June $1^{\text {st }}, 2020 /$ Accepted: August $20^{\text {th }}, 2020 /$ Online: September $12^{\text {th }}, 2020$

\section{INTRODUCTION}

Biofilms are protective communities of bacteria surrounded by extracellular polymeric substances (EPS) which are formed on surfaces, multicellular in nature, sessile and have a complex structure (Sauer et al., 2007). Majority of bacterial communities exist as biofilms and contribute to the survival of the bacteria. In nature, these biofilms act as a protection from adverse environmental conditions such as desiccation, chlorinated water, oxidative and osmotic stresses, as well as protects from protozoan predators (DEPas et al., 2014). In clinical settings, biofilm associated bacteria have also been noted to play a key role in infections particularly in chronic, nosocomial and medical device associated infections such as catheters.

In particular, E. coli existing as biofilms has been described as a major contributor to the disease causing ability of pathogenic strains, particularly as a major cause of recurrent urinary tract infections associated with medical devices such as catheters (Sharma et al., 2016). Biofilm formation has been associated with resistance to host immune responses, persistence and antimicrobial resistance (Adamus-Bialek et al., 2015; Bajpai et al., 2016). Potentially, all of these could play a role in increased levels of morbidity and mortality.

A recent study noted that an extensive focus in biofilm studies has been on laboratory rather than pathogenic strains (Schiebel et al., 2017). The study went on to assess a collection of pathogenic $E$. coli isolates and found a high association of biofilm-forming capabilities with a specific group of $E$. coli, the enteroaggregative $E$. coli (EAEC) using the Congo Red Agar method. Generally, however data on biofilm-forming potential of bacteria in Nigeria is sparse. An assessment of biofilm-forming potential of bacteria in this specific locale will be the first step in extensive biofilm 
studies aimed at elucidating pathogenesis and eventually instituting adequate control measures. This study was therefore aimed at analysing variations in biofilm-forming potential of E. coli from various sources in Port Harcourt, Nigeria.

\section{MATERIALS AND METHODS}

\section{A. Bacterial Isolates}

$E$. coli isolates used in this study were obtained from the bacterial collection of the Bacteriology group, Medical Microbiology Unit, University of Port Harcourt. These isolates consist of sixty previously characterized clinical (30) and non-clinical (30) Escherichia coli (Otokunefor et al., 2019).

\section{B. Detection of Biofilm-forming potential}

Biofilm-forming potential of isolates was determined using the Congo Red agar method (Ochi et al., 2020; Mathur et al., 2006). Purified bacterial isolates were subcultured on Congo red agar plates and incubated for 24 hours at $37^{\circ} \mathrm{C}$. The biofilm producing ability and the degree were determined as previously described. In brief, isolates producing black colonies are noted as biofilm formers, while non-biofilm formers produce red colonies. Biofilm producers were then further categorized as strong, moderate or weak biofilm formers according to the intensity of the colour of the colonies as previously described (Darwish and Asfour, 2013).

\section{Statistical Analysis}

Student t-test was used to analysis the data generated at $5 \%$ (0.05) significance level using IBM $^{\circledR}$ SPSS $^{\circledR}$ Statistics version 20.0 .

\section{RESULTS}

\section{A. Determination of biofilm-forming potential}

An assessment of the biofilm-forming potential of the $E$. coli isolates revealed that the majority of isolates, $(67 \%)$ had the potential to form biofilms (Figure 1). Non-clinical isolates however made up this majority of biofilm-forming isolates (Figure 2), though the difference was not statistically significant $(\mathrm{P}=0.2396$, two-tailed $\mathrm{T}$ test). Only $40 \%$ of isolates exhibiting biofilm-forming potential were from clinical sources. For both sets of isolates, the occurrence of isolates with biofilm-forming potential was higher than those without biofilm-forming potential (Figure 3 ). Unlike the non-clinical isolates where a $60 \%$ difference was noted in occurrence levels, for the clinical isolates the difference was only $6.8 \%$. This variation in biofilm forming ability between clinical and non-clinical bacteria was statistically significant with a $\mathrm{P}$ value of 0.030 .

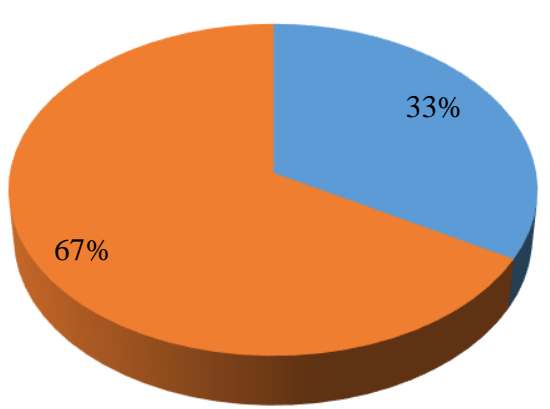

No Biofilm forming potential $\square$ Biofilm forming potential

Figure 1. Biofilm-forming potential of $E$. coli isolates

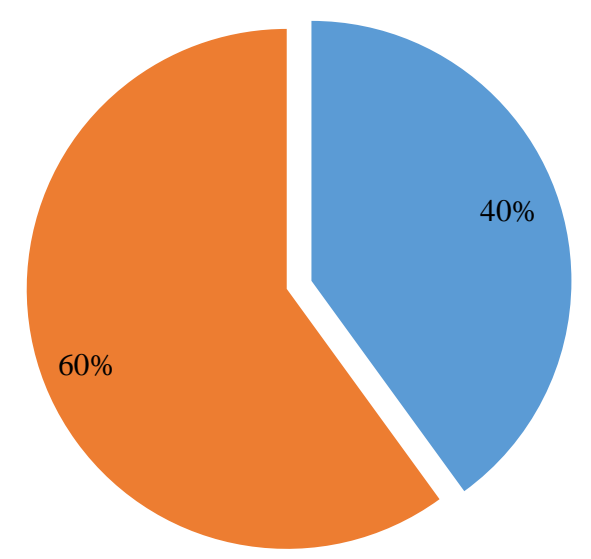

Clinical Biofilm formers $\square$ Non-Clinical Biofilm formers

Figure 2. Effect of isolate source on Biofilm-forming potential

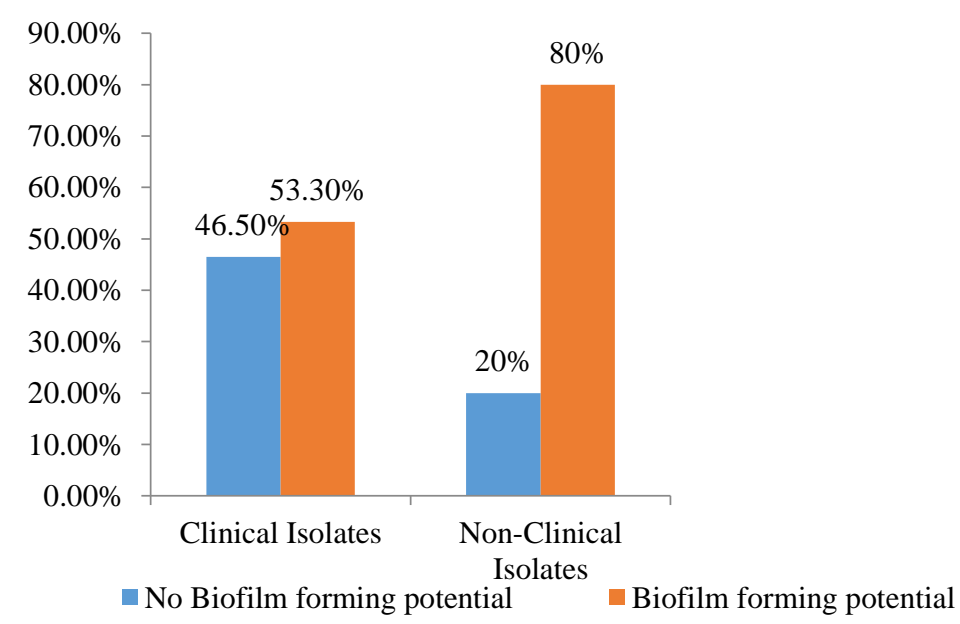

Figure 3. Variation of biofilm-forming potential based on source 
Despite the majority of isolates exhibiting biofilm-forming potential, the degree of this potential varied from weak to strong (Figure 4). Only non-clinical isolates exhibited strong biofilm-forming potential (Figure 5), while majority of both clinical and non-clinical isolates exhibited moderate biofilm-forming potential. A higher ratio of bacteria exhibiting a weak biofilm-forming potential was noted among the clinical rather than non-clinical isolates. These variations were however not statistically significant $(\mathrm{P}=$ $0.9651)$.
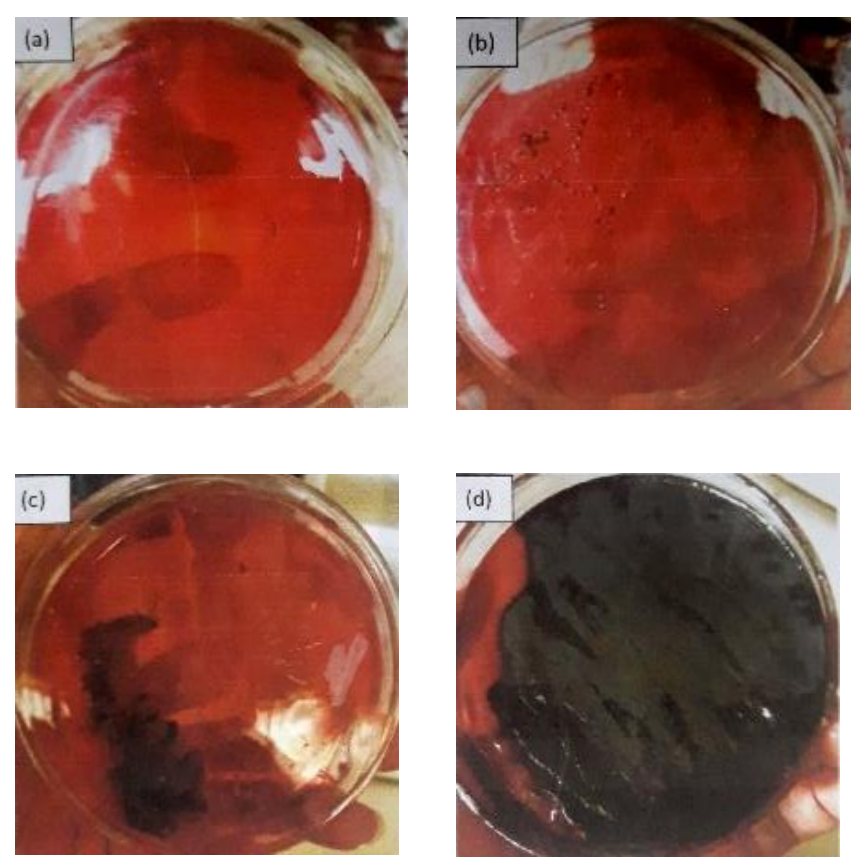

Figure 4. Variation in levels of biofilm-forming potential where (a.) no biofilm-forming potential; (b.) weak biofilm-forming potential; (c.) moderate biofilm-forming potential and (d.) strong biofilm-forming potential.

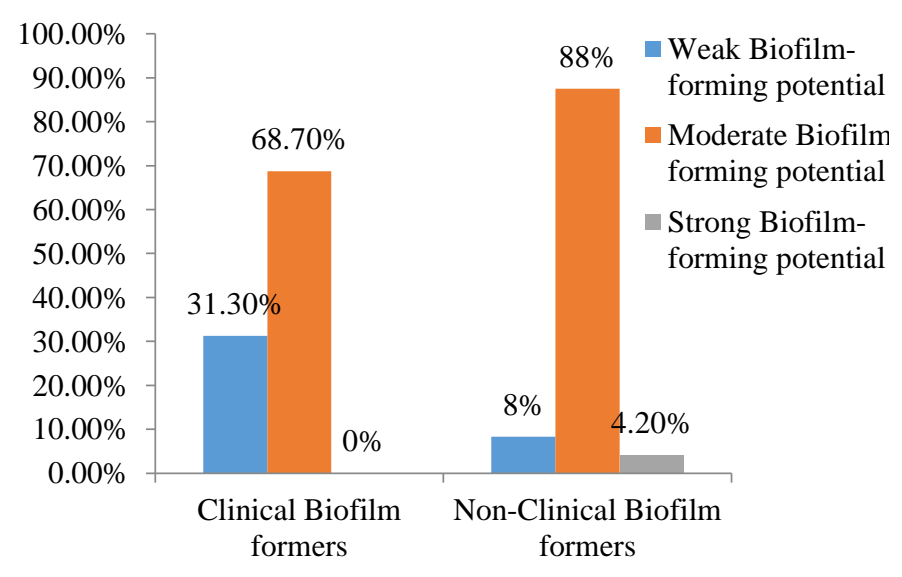

Figure 5. Effect of source on degree of biofilm-forming potential

\section{DISCUSSION}

Biofilm formation has long been associated with survival of bacteria both in nature and clinical settings. Studies have previously reported that the majority of bacteria are found in association with biofilms rather than free-living (Donlan and Costerton, 2002). Results of this present study noting a $67 \%$ occurrence of bacteria with biofilm-forming abilities agree with this. This study however noted a higher representation of non-clinical isolates among the isolates exhibiting biofilm-forming potential. In nature, biofilms are particularly advantageous as they play an essential role in the ability of the bacteria to survive (DEPas et al., 2014). Furthermore, environmental stresses have been reported to enhance the production of biofilms (Barbosa et al., 2013). Therefore, the $80 \%$ occurrence of isolates with biofilmforming potential among the non-clinical isolates is probably a reflection of adaptive mechanisms which these bacteria have adopted to avoid destruction.

Biofilms in clinical settings give the bacteria an increased chance of resistance. It may however not particular be essential in the initial colonization and invasion of the host which is the first step in pathogenesis. This lack of necessity of biofilms for the survival of clinical isolates is probably why a lower proportion of clinical isolates were found to possess biofilm-forming potential. The value associated with biofilm formation in clinical isolates is similar to the reported values of microbial infections associated with biofilm formation. Costerton et al., 1999 has been reported as describing a more than $50 \%$ association with bacterial infections (Hancock et al., 2007), while another review mentions a more than $60 \%$ association (Moscoso et al., 2009). Another study assessing biofilm formation in clinical isolates noted varying potential. Invasive isolates had the highest potential $(79 \%)$, followed by colonizers $(73 \%)$, with only $28 \%$ of commensals exhibiting this potential (Agarwal and Jain, 2013). Iorio and colleagues (Iorio et al., 2011) evaluating biofilm production in isolates from blood culture detected $85 \%$ of biofilm producers among their isolates. Banerjee and colleagues studying Enterobacteriaceae from healthy ducks noted biofilm-forming potential of $46.15 \%$ in Salmonella, $46.79 \%$ in E. coli and $70 \%$ in Klebsiella pneumoniae (Banerjee et al., 2019). The present study simply provides information on biofilm-forming potential of clinical isolates in general without a comparison with the specific role the isolates played in the clinical setting. This might contribute to the much lower figures observed.

Results of this study showing majority of the potential biofilm producing clinical isolates exhibiting moderate or weak biofilm producing abilities were similar to that of a previous study (Tajbakhsh et al., 2016). This study on biofilms in clinical isolates of $E$. coli observed that $81.25 \%$ of isolates exhibited either moderate or weak biofilm reaction. 


\section{V.CONCLUSION}

This work reports on a high level of association between $E$. coli isolates and the ability to form biofilms. Additionally, it highlights differences in the abilities of biofilm production between clinical and non-clinical isolates.

\section{REFERENCES}

Adamus-Białek, W., Kubiak, A., Czerwonka, G. (2015). Analysis of uropathogenic Escherichia coli biofilm formation under different growth conditions. Acta Biochimica Polonica, 62(4), 765 - 771.

Agarwal, A., Jain, A. (2013). Glucose \& sodium chloride induced biofilm production \& ica operon in clinical isolates of staphylococci. The Indian Journal of Medical Research, 138(2), 262 - 266.

Bajpai, T., Varma, M., Bhatambare, G. S., Pandey, M. (2016). Escherichia coli biofilms: Accepting the therapeutic challenges. International Journal of Health \& Allied Sciences, 5(4), $204-209$.

Banerjee, A., Bardhan, R., Chowdhury, M., Joardar, S. N., Isore, D. P., Batabayl, K., Dey, S., Sar, T. K., Bandyopadhyay, S., Dutta, T. K. Samanta, I. (2019). Characterization of beta-lactamase and biofilm producing Enterobacteriaceae isolated from organized and backyard farm ducks. Letters in Applied Microbiology, 69(2), 110 - 115.

Barbosa, J., Borges, S., Camilo, R., Magalhães, R., Ferreira, V., Santos, I., Silva, J., Almeida, G., Teixeira, P. (2013). Biofilm formation among clinical and food isolates of Listeria monocytogenes. International Journal of Microbiology, 524975.

Costerton, J. W., Stewart, P. S., Greenberg, E.P. (1999). Bacterial biofilms: a common cause of persistent infections. Science, 284(5418), 1318 1322.

Darwish, S.F., Asfour, H.A. (2013). Investigation of biofilm-forming ability in Staphylococci causing bovine mastitis using phenotypic and genotypic assays. The Scientific World Journal, 378492, 1-9.

DePas, W. H., Syed, A. K., Sifuentes, M., Lee, J. S., Warshaw, D., Saggar, V., Csankovszki, G., Boles, B.R., Chapman, M.R. (2014). Biofilm formation protects Escherichia coli against killing by Caenorhabditis elegans and Myxococcus xanthus. Applied and Environmental Microbiology, 80(22), $7079-7087$.

Donlan, R.M., Costerton, J.W. (2002). Biofilms: survival mechanisms of clinically relevant microorganisms. Clinical Microbiology Reviews, 15(2), 167-193.

Hancock, V., Ferrieres, L., Klemm, P. (2007). Biofilm formation by asymptomatic and virulent urinary tract infectious Escherichia coli strains. FEMS Microbiology Letters, 267(1), 30 - 37.

Iorio, N. L., Lopes, A. P., Schuenck, R. P., Barcellos, A. G., Olendzki, A. N., Lopez, G. L., dos Santos, K. R. (2011). A combination of methods to evaluate biofilm production may help to determine the clinical relevance of Staphylococcus in blood cultures. Microbiology and Immunology, 55(1), 28 - 33.

Mathur, T., Singhal, S., Khan, S., Upadhyay, D. J., Fatma, T., Rattan, A. (2006). Detection of biofilm formation among the clinical isolates of staphylococci: an evaluation of three different screening methods. Indian Journal of Medical Microbiology, 24(1), 25 - 29.

Moscoso, M., García, E., López, R. (2009). Pneumococcal biofilms. International Microbiology, 12, 77 - 85.

Otokunefor, K., Osogho, V.O., Nwankwo C.P. (2019). Escherichia coli as possible agents of spread of multidrug resistance. Annals of Science and Technology, 4(1), 16-21.

Ochi, P., Otokunefor, K., Abu, G. (2020). Diversity of biofilm producing bacteria in a drinking water distribution system in a sub-urban community in South-South Nigeria. World Journal of Biomedical Research, 7(1), 8 - 14 .

Sauer, K., Rickard, A.H., Davies, D.G. (2007). Biofilms and Biocomplexity. Microbe, 2, 347 - 353.

Schiebel, J., Böhm, A., Nitschke, J., Burdukiewicz, M., Weinreich, J., Ali, A., Roggenbuck, D., Rödiger, S., Schierack, P. (2017). Genotypic and phenotypic characteristics associated with biofilm formation by human clinical Escherichia coli isolates of different pathotypes. Applied and Environmental Microbiology, 83(24), e01660-17.
Sharma, G., Sharma, S., Sharma, P., Chandola, D., Dang, S., Gupta, S., Gabrani, R. (2016). Escherichia coli biofilm: development and therapeutic strategies. Journal of Applied Microbiology, 121(2), 309 319.

Tajbakhsh, E., Ahmadi, P., Abedpour-Dehkordi, E., Arbab-Soleimani, N., Khamesipour F. (2016). Biofilm formation, antimicrobial susceptibility, serogroups and virulence genes of uropathogenic $E$. coli isolated from clinical samples in Iran. Antimicrobial Resistance \& Infection Control, 5(1), 11. 